\title{
High-resolution genomic analysis: the tumor-immune interface comes into focus
}

\author{
Jonathan J Havel ${ }^{1}$ and Timothy A Chan ${ }^{1,2^{*}}$ \\ Please see related article: http://dx.doi.org/10.1186/s13059-015-0620-6
}

\begin{abstract}
A genomic analysis of heterogeneous colorectal tumor samples has uncovered interactions between immunophenotype and various aspects of tumor biology, with implications for informing the choice of immunotherapies for specific patients and guiding the design of personalized neoantigen-based vaccines.
\end{abstract}

Immunotherapy is a promising new approach for treating human malignancies. Approximately $20 \%$ of melanoma and lung cancer patients receiving immune checkpoint inhibitors show responses [1,2]. Current major challenges include identification of patients most likely to respond to specific therapies and elucidation of novel targets to treat those who do not. To address these problems, a detailed understanding of the dynamic interactions between tumors and the immune system is required. In a new study, Zlatko Trajanoski and colleagues [3] describe a powerful approach to dissecting these issues through high-resolution analysis of patient genomic data. This study represents a significant advance over previous work from this group, which defined 28 immune-cell-type gene expression signatures and identified specific cell types as prognostic indicators in colorectal cancer (CRC) patients [4]. Here, the authors [3] integrate genomic analyses of CRC tumor molecular phenotypes, predicted antigenicity (called the 'antigenome'), and immune-cell infiltration derived from multiple independent cohorts to gain refined insights into tumor-immune system interactions.

\footnotetext{
* Correspondence: chant@mskcc.org

${ }^{1}$ Human Oncology and Pathogenesis Program, Memorial Sloan Kettering Cancer Center, New York, NY 10065, USA

${ }^{2}$ Department of Radiation Oncology, Memorial Sloan Kettering Cancer Center, New York, NY 10065, USA
}

\section{Not all tumor-infiltrating lymphocytes are created equal}

Past studies have used immune-staining techniques to determine associations between a limited set of infiltraichment analysis (GSEA) of immune cell expression signatures to ascertain associations of 28 immune-cell populations with patient survival and tumor molecular phenotypes. Effector memory $\mathrm{CD}^{+}$and $\mathrm{CD}^{+}{ }^{+} \mathrm{T}$ cells, natural killer cells, and activated dendritic cells are significantly associated with improved overall survival. Interestingly, although the authors' previous work found no significant prognostic value of regulatory $\mathrm{T}$ cells (Tregs) or myeloid-derived suppressor cells (MDSCs) [4], negative associations of these cell types with overall survival are among the strongest relationships observed in the current study. It is possible that variations in sample collection and preparation may have contributed to this discrepancy. The conclusions, supported by the numerous animal studies demonstrating the importance f cell-mediated immunosuppression, are substantially this study.

Another important observation is the association of specific immune cell subsets with CRC tumor stage and molecular phenotypes as classified by mutation rate, microsatellite instability, and methylation status. This knowledge will be crucial in determining which types of immunotherapy are most likely to benefit individual patients. Interestingly, although hypermutated microsatelliteunstable tumors show strong enrichment of adaptive immune cells, similar enrichment is notably lacking in the small population of hypermutated microsatellitestable tumors. This raises an intriguing question of whether and how microsatellite instability/mismatch repair may independently shape immune responses. 
Furthermore, Trajanoski and colleagues [3] find that tumor-infiltrating lymphocytes transition from an adaptive to an innate immunophenotype with increasing tumor stage. This raises an interesting issue of whether immunotherapies that depend on the adaptive immune response can be effective in later stage CRC tumors.

\section{Diversity of tumor antigens}

In addition to characterizing immune components involved in tumor immune responses, it is equally important to identify and understand the tumor-associated antigens that elicit these responses, called the 'antigenome'. The authors [3] analyze RNA-seq and genomic data to identify two types of tumor antigens in CRC - non-mutated cancer germline antigens that are aberrantly overexpressed, and neoantigens, which are generated from non-synonymous somatic mutations. Importantly, the authors [3] find that cancer-germline antigens are highly shared among patients and are independent of molecular and immune phenotype. In contrast, neoantigens are enriched in the hypermutated microsatellite-unstable phenotype tumors and rarely shared among patients. These results imply a heightened importance of neoantigens in comparison to cancer-germline antigens [7]. In addition, similar analy- tical methods have recently been applied to identify functional neoantigens in human melanoma and cholangiocarcinoma [8-10]. An emerging theme of these studies is that the in vitro validation rate for predicted neoantigens is relatively low; however, it is unclear whether this is due to limited sensitivity of functional assays or epigenetic silencing to circumvent immunoediting, or whether the number of immunogenic neoantigens is in fact small. Interestingly, Trajanoski and colleagues [3] find a modest yet significant decrease in neoantigen frequency with increasing tumor stage. Considering the concomitant decrease in adaptive immune cell infiltration, it is tempting to speculate that this phenomenon reflects immunoediting of critical neoepitopes during tumor progression. Furthermore, the authors find an association, albeit not statistically significant, between increased neoantigen burden and improved patient survival. This finding complements a recent report [9] showing that whereas neoantigen burden roughly predicts survival of anti-CTLA-4-treated melanoma patients, a collection of consensus neoepitope motifs is strongly associated with patient survival. It will be interesting to see if future studies can determine the effect of CRC neoantigen burden in the setting of immunotherapy, and answer the questions of whether an

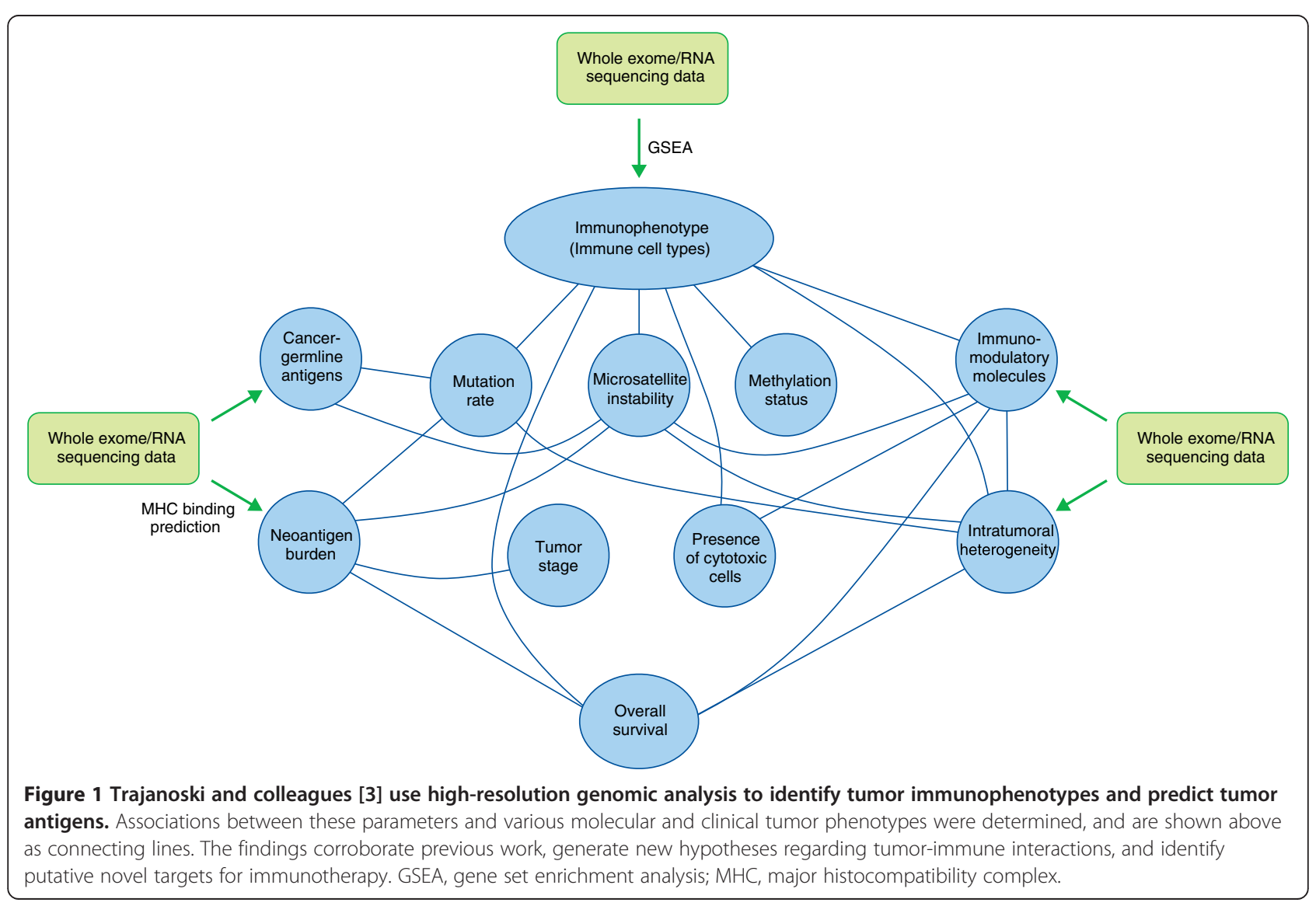


analogous signature of prognostic neoepitope motifs exists for CRC, and whether there are any similarities between substring signatures of different tumor types.

\section{Tumor escape mechanisms and new targets - one size does not fit all}

All tumors evade immune system destruction through any of numerous possible mechanisms. Knowing which escape mechanism(s) to target in specific tumors is critically important for successful immunotherapy. The authors [3] analyze immune infiltrate composition and immune modulatory molecule expression in relation to molecular CRC tumor phenotypes. Importantly, they find that in hypermutated tumors, immunoinhibitory molecules such as CTLA-4 and PD-1 are upregulated, whereas immunosuppressive cells such as Tregs and MDSCs are depleted. This finding confirms previous studies demonstrating that increased mutational burden is associated with response to anti-CTLA-4 and anti-PD-1 therapies [9]. On the other hand, non-hypermutated tumors show increased immunosuppressive cell infiltration and downregulation of immunoinhibitory molecules [3], highlighting the need to develop Treg- and MDSC-directed therapies. Finally, the authors use two modeling techniques to identify BCMA (TNFRSF17) and CCR8 as putative novel targets for immunotherapy. CCR8 is identified as a determinant of tumor immunogenicity, and both molecules are positively associated with patient survival. It remains an intriguing question whether agonists of these molecules can improve response to immunotherapies.

\section{Conclusions}

Trajanoski and colleagues [3] describe a fascinating new application of genomic analysis in heterogeneous tumor samples to uncover interactions among the immunophenotype and various aspects of CRC tumor biology. Extension of these analyses will help inform the choice of immunotherapies for specific patients and guide the design of personalized neoantigen-based vaccines. Although some of the authors' findings have been demonstrated previously by other methods, the fact that all conclusions here are derived from computational analysis demonstrates the power and efficiency of such an approach (Figure 1). It will be of great value to apply these methods to datasets from other tumor types and in the context of response to immunotherapies. By using innovative genomic analyses, Trajanoski and colleagues [3] have shed new light on the clandestine world of immune cell-tumor interactions and bring us a step closer toward implementation of personalized cancer immunotherapies.

\footnotetext{
Abbreviations

CRC: Colorectal cancer; CTLA-4: Cytotoxic T-lymphocyte-associated protein 4; GSEA: Gene set enrichment analysis; MDSCs: Myeloid-derived suppressor cells; PD-1: Programmed cell death protein 1; Treg: Regulatory T cell.
}

\section{Competing interests}

The authors declare that they have no competing interests.

Published online: 31 March 2015

\section{References}

1. Topalian SL, Hodi FS, Brahmer JR, Gettinger SN, Smith DC, McDermott DF, et al. Safety, activity, and immune correlates of anti-PD-1 antibody in cancer. N Engl J Med. 2012;366:2443-54.

2. Maio M, Grob JJ, Aamdal S, Bondarenko I, Robert C, Thomas L, et al. Five-year survival rates for treatment-naive patients with advanced melanoma who received ipilimumab plus dacarbazine in a phase III trial. J Clin Oncol. 2015. doi:10.1200/JCO.2014.56.6018.

3. Angelova M, Charoentong P, Hackl H, Fischer ML, Snajder R, Krogsdam AM, et al. Characterization of the immunophenotypes and the antigenomes reveal distinct tumor escape mechanisms and novel targets for immunotherapy of colorectal cancers. Genome Biol. 2015;16:620.

4. Bindea G, Mlecnik B, Tosolini M, Kirilovsky A, Waldner M, Obenauf AC, et al. Spatiotemporal dynamics of intratumoral immune cells reveal the immune landscape in human cancer. Immunity. 2013;39:782-95.

5. Galon J, Costes A, Sanchez-Cabo F, Kirilovsky A, Mlecnik B, Lagorce-Pages C, et al. Type, density, and location of immune cells within human colorectal tumors predict clinical outcome. Science. 2006;313:1960-4.

6. Llosa NJ, Cruise M, Tam A, Wicks EC, Hechenbleikner EM, Taube JM, et al. The vigorous immune microenvironment of microsatellite instable colon cancer is balanced by multiple counter-inhibitory checkpoints. Cancer Discov. 2015:5:43-51.

7. Lennerz V, Fatho M, Gentilini C, Frye RA, Lifke A, Ferel D, et al. The response of autologous T cells to a human melanoma is dominated by mutated neoantigens. Proc Natl Acad Sci U S A. 2005;102:16013-18.

8. van Rooij N, van Buuren MM, Philips D, Velds A, Toebes M, Heemskerk B, et al. Tumor exome analysis reveals neoantigen-specific T-cell reactivity in an ipilimumab-responsive melanoma. J Clin Oncol. 2013;31:e439-42.

9. Snyder A, Makarov V, Merghoub T, Yuan J, Zaretsky JM, Desrichard A, et al. Genetic basis for clinical response to CTLA-4 blockade in melanoma. N Engl J Med. 2014;371:2189-99.

10. Tran E, Turcotte S, Gros A, Robbins PF, Lu YC, Dudley ME, et al. Cancer immunotherapy based on mutation-specific CD4+ T cells in a patient with epithelial cancer. Science. 2014;344:641-5. 